\title{
EDITORIAL
}

\section{Evidence-based practice in the evaluation and treatment of sexual offenders}

\author{
John M Bradford ${ }^{1,2,3}$, Abdullah H Alqahtani ${ }^{2,4}$, Andrew T Olagunju ${ }^{2}$
}

\author{
St. Joseph's Healthcare Hamilton, Forensic \\ Psychiatry Program, Hamilton, Canada \\ 2 McMaster University, Department of Psychiatry \\ and Behavioural Neurosciences, Hamilton, Canada \\ 3 University of Ottawa, Department of Psychiatry, \\ Ottawa, Canadda \\ 4 Department of Psychiatry, College of Medicine, \\ Imam Abdulrahman Bin Faisal University,Dammam, \\ Saudi Arabia
}

This editorial addresses evidence based medical practice in forensic psychiatry and particularly in the field of paraphilia. John Bradford is a Professor in the Department of Psychiatry and Behavioural Neurosciences, McMaster University. He is an Emeritus Professor at the University of Ottawa where he was a founder of the Royal Ottawa Institute of Mental Health Research. He is a Founder of Forensic Psychiatry, granted by the Royal College of Physicians and Surgeons of Canada. Abdullah Alqahtani is an Assistant Professor and Consultant Psychiatrist at King Fahd University Hospital, Imam Abdulrahman Bin Faisal University, Dammam, Saudi Arabia. He is currently completing a clinical fellowship in forensic psychiatry at McMaster University - St. Joseph's Healthcare Hamilton. Andrew Olagunju is an academic psychiatrist with a Senior Lecturer position at the College of Medicine, University of Lagos, Nigeria. He is also completing a clinical fellowship at McMaster University - St. Joseph's Healthcare Hamilton.

One of the significant shifts in medicine in the last two decades is the introduction of evidence-based practice, characterized by the integration of clinical expertise with best available evidence from systematic research in making a clinical decision $[1,2]$. Given the pressure to adopt this approach in all medical disciplines, evidencebased practice is in vogue in medicine and a hot topic in forensic psychiatry. For instance, analogous to evidence-based practice, psychiatric expert testimony is required to be objective and scientifically based, and the Supreme Courts of Canada and USA in relevant case laws have established a scientific basis for the reliability of psychiatric expert evidence [3].
The US Supreme Court held that the minimum requirement for admissibility of scientific evidence (and the weight once admitted) be based on its scientific validity as described in Daubert [4]. In a similar case, the Canadian Supreme Court, in R. v. Mohan, noted that four factors control the admissibility of expert evidence: relevance, the necessity in assisting the trier of fact, the absence of any exclusionary rule, and the proper qualification of the expert [5]. Given the preceding decisions, there is an apparent burden to show that the theory or technique underlining evidence is tested, peer-reviewed, has a known error rate or standard of reference, and is generally accepted within the scientific community $[4,6,7]$. In fact, it is often said that an expert opinion is only as good as the factual foundation on which it is premised [7]. This puts the responsibility on the forensic psychiatrist witness to be aware of the evidence-based practice in their roles within the criminal justice system and those interfacing with the civil mental health system.

As simple as it might sound, there are caveats on how evidence-based medicine applies to forensic psychiatric practice because "one medicine or rule for all" does not always hold [8]. This is because some aspects of forensic psychiatric practice have a stronger scientific basis compared to others, and clearly fit the definition of evidence-based medicine. A "stand-alone" application of evidence-based practice to all aspects of forensic psychiatry, especially when it is devoid of clinical judgment, can be counter-intuitive, too reductionist, over-dependent on clinical trials, and would not fit all the complexities of medico-legal cases $[7,9]$. The assessment and treatment of sexual offenders provide a good example of a strong scientific basis 
and meet the definition of evidence-based medicine in many respects, as defined by Sackett et al. [10].

When dealing with a sexual offender, the criminal justice system needs scientific evidence regarding the diagnostic criteria for paraphilia, the risk of recidivism, and the possible treatments that would mitigate that risk. All of these areas have been the subject of considerable research and would fit the definition of evidence-based medicine $[1,10]$. In this editorial, we model evidence-based practice by parsing the best available evidence for the assessment and treatment of sexual offenders while highlighting some limitations. We also revisit the issue of the complementarity of clinical acumen and knowledge derived from empirical research in forensic psychiatry.

\section{Evidence-Base Practice in Assessment and Diagnosis of Paraphilia}

When assessing a sexual offender, it is important to highlight the psychopathological characteristics of the individual's sexual behaviour in order to understand why it deviated from the norm. Studies related to human sexuality have provided an understanding of "anomalous psychosexual phenomenon," while evidence-based forensic psychiatric practice has specifically allowed refinement of diagnostic assessments and nosology of the paraphilias. For example, the successive revisions of the Diagnostic and Statistical Manual of Mental Disorders (DSM-5), now in its fifth edition, led to the historical delisting of homosexuality and modification in the diagnosis of paraphilia as new scientific evidence emerged [11].

According to the DSM-5, paraphilia is characterized by recurrent, intense sexual urges, fantasies, or behaviors that involve unusual objects, activities, or situations and cause clinically significant distress or impairment in social, occupational, or other important areas of functioning [11]. The paraphilias consist of exhibitionism, fetishism, frotteurism, pedophilia, sexual masochism, sexual sadism, transvestic fetishism, voyeurism and paraphilia not otherwise specified (this includes telephone scatalogia; necrophilia; partialism; zoophilia; coprophilia; klismaphilia and urophilia). Although by definition, all of the paraphilias can be diagnosed with only the presence of deviant sexual fantasies, clearly the behavior of some paraphilias require physical contact with an adult or child, or an animal in zoophilia and a corpse in necrophilia. In the other paraphilias, the behavior does not require contact with another person. The former group is regarded as the "hands-on" paraphilias, while the latter group is regarded as the "hands-off" paraphilias. This classification is not to be confused with victimization, whereby clearly the victims of voyeurism, exhibitionism and telephone scatalogia, for example, can be traumatized by the experience. When considered as a group of diagnoses in psychiatry, there are only a small number of the paraphilias that would clearly fit the definition of being sexually violent, and sexual violence is mostly related to "hands-on" paraphilias. That said, there is considerable comorbidity between the paraphilias, with significant crossover in any given individual between "hands-on" and "hands-off" paraphilias [12].

The phenomenon of comorbidity emphasizes the need for a careful diagnostic evaluation. This type of assessment is based on the pathognomonic feature of all paraphilias or sexual deviations, which is deviant sexual arousal [13]. This then formed the basis for the objective measure of sexual arousal, which would then define the sexual preference of the individual and allow for objective diagnosis of the paraphilia or sexual deviation. Deviant sexual preference, in theory, drove deviant sexual behavior and this was responsible for sexual offending in the majority of cases.

The objective measurement of sexual arousal in a laboratory setting also provided an independent measurement of the reported sexual preference by the sexual offender or persons suffering from a paraphilia. This is made possible by the measurement of penile tumescence in a laboratory setting as an objective measure of sexual preference [14]. This objective test formed a fundamental basis to the comprehensive assessment of sexual offenders. Although there is no complete stand- 
ardized approach to the evaluation of sexual offenders in specialized centers, a typical approach includes a forensic psychiatric diagnostic and evaluative clinical examination, a detailed psychiatric history, mental status examination to diagnose associated psychiatric conditions and general medical conditions. In addition, specific assessment for deviant sexual behavior consists of sex hormone profile, a variety of sexual questionnaires, and objective measures of sexual interest such as penile tumescence testing or visual reaction time [15]. The sex hormone profile consists of free and total testosterone (Free $\mathrm{T}$ and Total $\mathrm{T})$; follicle stimulating hormone (FSH); luteinizing hormone ( $\mathrm{LH})$; estradiol; prolactin; and progesterone. The sex hormone profile is essential to form the diagnostic basis for conditions affecting sexual endocrinology that may be associated with paraphilias, such as Klinefelter syndrome. It also establishes the baseline readings for pharmacological treatment intervention. The sexual questionnaires are usually selfreport questionnaires measuring overall sexual performance, drug and alcohol usage, sexual drive measures, general measures of impulsivity, measures of aggression, quantitative and qualitative measures of sexual fantasy, a detailed sexual behaviors inventory, some measure of deception, and measurement of cognitive distortions. Physiological measures of sexual preference complete the overall comprehensive assessment [15]. Despite the scientific basis to penile tumescence testing (penile plethysmography - PPG), there has been a considerable amount of criticism, and to a certain extent controversy, about using these techniques to make a diagnosis, as well as their use in forensic settings. The main issue is the lack of standardization of the procedures used and the lack of a standardized stimulus set. Another area of criticism for PPG testing is the lack of consensus as to the appropriate content and method of delivery of each stimulus; the usual delivery is a videotape, slides and audiotapes.

\section{Evidence-Based Practice in Risk As- sessments}

Sexual offense recidivism risk is calculated through risk assessment instruments such as the Static 99 [16], and often Psychopathy is measured through the Hare Psychopathy Checklist [17]. The importance of sexual arousal testing is emphasized by deviant sexual preference being amongst the strongest predictors of sexual offense recidivism [18]. Although a full discussion of risk assessment and risk assessment instruments is beyond the scope of this paper, the Hare Psychopathy Checklist forms an important part of the evidencebased practice in the assessment of sexual offenders as it forms an important part of the prediction of future sexual offense recidivism. The concept of psychopathy has developed historically and is the product of extensive clinical research [18]. The concept as operationalized in the Hare Psychopathy Checklist (PCLR) has proven to be the most reliable means of identifying psychopathic traits [17]. It also has become the most important tool in assessing psychopathy in forensic psychiatric situations such as risk assessment but is also proved to be one of the most important measurements in the prediction of recidivism [18]. The performance of other risk assessment instruments for predicting sexual offense recidivism have also been significantly researched and have significant predictive validity $[16,18]$. The extensive recidivism studies, particularly looking at violent sexual offenders, have participated in the evidence-based testimony in Dangerous Offender hearings in Canada and in Sexually Violent Predator hearings in the United States.

\section{Evidence-Based Practice in Treatment of Sexual Offenders}

The pharmacological treatment of sexual offenders is based on well-established studies on the neurobiology and neuropharmacology of sexual behavior in both human and animal subjects [19]. In fact, the neurobiology and neuropharmacology of sexual behavior is far better understood compared to the neuropharmacology and neurobiology of major psychiatric disorders such as Mood Disorders and Schizo- 
phrenia [19]. Historically, surgical castration was widely used as an intervention to treat sexual offenders, and various studies reported dramatic decreases in sexual offense recidivism based on this intervention. Pharmacological treatments have been developed using the same principle of intervention, that is, the reduction of total and free testosterone in the endocrine system, significantly reducing sexual drive and consequently sexual behavior, including deviant sexual behavior. The pharmacological treatments provided a reversible intervention compared to surgical castration. Pharmacological intervention includes three main categories: Selective serotonin reuptake inhibitors (SSRIs); Hormonal agents such as medroxyprogesterone acetate (MPA) and luteinizing hormone-releasing hormone (LHRH) agonists (leuprolide acetate and goserelin acetates are the most commonly used); and Antiandrogens (cyproterone acetate (CPA) is the most widely used) [19].

In addition to the neuropharmacology and neurobiology of these pharmacological agents being well understood, it has also formed the basis of a treatment algorithm for the pharmacological treatment of the paraphilias and sexual offenders [19]. This algorithm starts with all persons requiring treatment receive psychological treatments in the form of cognitive behavioral therapy and relapse prevention treatment (level one). This is followed by various levels of pharmacological treatment starting with selective serotonin reuptake inhibitors (SSRIs), a non-hormonal treatment and the least intrusive of pharmacological interventions (level two). Next is hormonal (MPA) or antiandrogen (CPA) treatments given orally (level three); this is followed by a combination of an oral antiandrogen (CPA) or hormonal therapy (MPA) given in conjunction with an SSRI (level four). This has been followed by intramuscular anti-

\section{References}

1. Guyatt $\mathrm{GH}$. Evidence-based medicine. ACP J Club 1991;114:(suppl 2) A16.

2. Sackett DL. Evidence-based medicine. Seminars in Perinatology 1997;21(1):3-5. androgen (CPA) or hormonal (MPA) treatment (level five). Finally, there is $\mathrm{LHRH}$ agonist treatment resulting in a pharmacological castration using leuprolide acetate or goserelin acetate (level six). The decision to move from one level of the algorithm through to the next level, and ultimately to level six, is based on a severity scale.

\section{Limitations}

Evidence-based practice can impose some limitations on forensic psychiatry. Scientific validity is a necessary but not sufficient precondition in determining if the evidence is admissible in court. Presumably, evidence-based medicine will inform the court as to the scientific validity of the evidence, while admissibility and adjudication of a case in point rests with the court. It is also not clear how much of patients' best interests as well as physician education play into evidence-based practice. In the same vein, a pragmatic use of clinical trial evidence seems essential to ensure the validity of the therapies used; for example, no randomized controlled studies of the efficacy of SSRIs in the treatment of the paraphilias have been published to date.

\section{Recommendation}

The science underpinning assessment and treatment of sexual offenders continues to evolve, and forensic psychiatric professionals are expected to be cognizant of this recent scientific evidence. Despite the complexities of medico-legal cases, the evidence-based practice remains an essential tool to continue to improve medical knowledge of sexual offenders.
3. Glancy GD, Bradford JM. The admissibility of expert evidence in Canada. J Am Acad Psychiatry Law 2007;35(3):350-6.

4. Daubert v. Merrell Dow. Pharmaceuticals, Inc. 509 U.S. 579 (1993)y. (accessed December 20, 2018) 
5. R. v. Mohan. [1992] 2 S.C.R. 9. (accessed December 20, 2018)

6. R. v. J. (J.-L.). [1999] 130 C.C.C. (3d) 541 (Que. C.A.). . (accessed December 20, 2018)

7. Schneider RD. Commentary: evidence-based practice and forensic psychiatry. J Am Acad Psychiatry Law 2009;37(4):503-8.

8. Borenstein D. Evidence-based psychiatry. Psychiatric News 2001;36:3.

9. Glancy GD, Saini M. The confluence of evidence-based practice and Daubert within the fields of forensic psychiatry and the law. J Am Acad Psychiatry Law 2009;37(4):438-41.

10. Sackett DL, Rosenberg WM, Gray JA, Haynes RB, Richardson WS. Evidence based medicine: what it is and what it isn't. Clin Orthop Relat Res 2007;455:3-5.

11. American Psychiatric Association. Diagnostic and Statistical Manual of Mental Disorders. $5^{\text {th }}$ edition. Washington DC, USA: American Psychiatric Publishing, 2014.

12. Bradford JM, Boulet J, Pawlak A. The paraphilias: a multiplicity of deviant behaviours. Can J Psychiatry 1992;37(2):104-8.

13. Bancroft JH, Jones HG, Pullan BR. A simple transducer for measuring penile erection with comments on its use in the treatment of sexual disorders. Behav Res Ther 1966;4(3):239-41

14. Fedoroff JP, Kuban M, Bradford JM. Laboratory measurements of penile response in the assessment of sexual interests. In: Saleh F, Grudzinskas A, Bradford J, Brodsky (Eds). Sex Offenders: Identification, risk assessment, treatment and legal issues. New York: Oxford University Press; 2009.

15. Bourget D, Bradford JM, John MW. Evidential Basis for the Assessment and Treatment of Sex Offenders. Brief Treat Crisis Interv 2008;8(1):130-146.

16. Hanson RK, Thornton D. Improving risk assessment of sex offenders: A comparison of three acturial scales. Law Hum Behav 2000;24:119-136.

17. Hare RD (Ed). The Hare Psychopathy Checklist Revised (PCL-R). 2nd Ed. Toronto, ON: MultiHealth Systems Inc. 2003.

18. Hanson RK, Bussiere MT. Predicting relapse: a meta-analysis of sexual offender recidivism studies. J Consult Clin Psychol 1998;66(2):34862.

19. Bancroft J. The endocrinology of sexual arousal. J Endocrinol 2005;186(3):411-27.

\section{Corresponding author}

John Bradford, Forensic Psychiatry Program, St. Joseph's Healthcare Hamilton, Hamilton ON L9C 0E3, Canada - email: jbradford@stjosham.on.ca 